\title{
High Accuracy Arithmetic Average Discretization for Non-Linear Two Point Boundary Value Problems with a Source Function in Integral Form
}

\author{
Ranjan K. Mohanty, Deepika Dhall \\ Department of Mathematics, Faculty of Mathematical Sciences, University of Delhi, Delhi, India \\ E-mail: rmohanty@maths.du.ac.in \\ Received June 17, 2011; revised June 30, 2011; accepted July 6, 2011
}

\begin{abstract}
In this article, we report the derivation of high accuracy finite difference method based on arithmetic average discretization for the solution of $u^{\prime \prime}=F\left(x, u, u^{\prime}\right)+\int_{0}^{1} K(x, s) \mathrm{d} s, 0<x<1,0<s<1$ subject to natural boundary conditions on a non-uniform mesh. The proposed variable mesh approximation is directly applicable to the integro-differential equation with singular coefficients. We need not require any special discretization to obtain the solution near the singular point. The convergence analysis of a difference scheme for the diffusion convection equation is briefly discussed. The presented variable mesh strategy is applicable when the internal grid points of the solution space are both even and odd in number as compared to the method discussed by authors in their previous work in which the internal grid points are strictly odd in number. The advantage of using this new variable mesh strategy is highlighted computationally.
\end{abstract}

Keywords: Variable Mesh, Arithmetic Average Discretization, Non-Linear Integro-Differential Equation, Diffusion Equation, Simpson's $\frac{1}{3}$ Rd Rule, Singular Coefficients, Burgers' Equation, Maximum Absolute Errors

\section{Introduction}

We consider the non-linear differential equation with a source function in integral form:

$$
u^{\prime \prime}=F\left(x, u, u^{\prime}\right)+\int_{0}^{1} K(x, s) \mathrm{d} s, 0<x, s<1 .
$$

The two point boundary conditions associated with (1) are given by:

$$
u(0)=\gamma_{0}, u(1)=\gamma_{1}
$$

where $\gamma_{0}, \gamma_{1}$ are finite constants. We assume that $K(x, s)$ is a real valued function of both variables in the range $0 \leq x, s \leq 1$.

Let

$$
I(x)=\int_{0}^{1} K(x, s) \mathrm{d} s
$$

and

$$
F\left(x, u, u^{\prime}\right)+I(x)=G\left(x, u, u^{\prime}\right)
$$

Then we may re-write (1) as

$$
u^{\prime \prime}=G\left(x, u, u^{\prime}\right), 0<x<1
$$

Keller [1] has given the conditions under which the differential Equation (3) together with the boundary conditions (2) has a unique solution. We assume that these conditions are satisfied in the problem that we are considering. In addition, we assume that $u(x) \in C^{6}[0,1]$ and $K(x, s) \in C^{4}[0,1]$.

Many physical problems from fluid mechanics, fluid dynamics, elasticity, magneto-hydrodynamics, plasma dynamics, oceanography, biological model, boundary layer theory, ...etc are described mathematically by nonlinear integro-differential equations. Davis and Rabinowitz [2], Philips [3], Linz [4], Lakshmikantham and 
Rao [5], Atkinson [6], and Agarwal and O'Regan [7] have discussed various techniques for numerical integration and methods for approximate solution of integrodifferential equations and their applications to various physical models. Most of the nonlinear differential equations cannot be solved analytically. So it is required to obtain efficient numerical methods. Jain et al. [8] have discussed variable mesh methods for the solution of two point nonlinear boundary value problems; however, their methods are not applicable to differential equations with singular coefficients. In recent years, Mohanty et al. ([912]) have discussed a family of third order variable mesh methods for the solution of two point non-linear boundary value problems and obtained convergent solution for singular problems. More recently, Mohanty and Dhall [13] have proposed a three-point third order variable mesh method for the solution of non-linear integro-differential Equation (1), which is applicable only when the internal grid points of the solution region are odd in number. In this paper, we propose an efficient third order variable mesh method based on arithmetic average discretization for the solution of non-linear integro-differential Equation (1), which is applicable when the internal grid points of the solution region are both odd and even in number. In next section, we give mathematical details of the method. In Section 3, we discuss the application of the proposed method to an integro-differential equation with singular coefficients and study the convergence analysis. In Section 4, we give numerical results to justify the utility of the proposed new strategy. Final remarks are given in Section 5.

\section{Mathematical Details of the Discretization}

We discretize the solution region $[0,1]$ with the nonuniform mesh such that $0=x_{0}<x_{1} \cdots<x_{N+1}=1$. Let $h_{k+1}=x_{k+1}-x_{k}>0$ be the variable mesh size in $x$-direction, where $k=0(1) N+1$. Grid points are given by $x_{i}=x_{0}+\sum_{k=1}^{i} h_{k}, i=1(1) N+1$. The mesh ratio is $\sigma_{k}=\left(h_{k+1} / h_{k}\right)>0$. When $\sigma_{k}=1$, then it reduces to the constant mesh case. The off-step points are defined by $x_{k+\frac{1}{2}}=x_{k}+\frac{\sigma_{k} h_{k}}{2}$ and $x_{k-\frac{1}{2}}=x_{k}-\frac{h_{k}}{2}$ etc. Let the exact solution of $u(x)$ at the grid point $x_{k}$ be denoted by $U_{k}=u\left(x_{k}\right)$ and $u_{k}$ be the approximate value of $U_{k}$.

Let us construct a numerical method for evaluating the integral $\int_{0}^{1} \phi(x) \mathrm{d} x$, where $\phi(x)$ is a real-valued continuous function in $[0,1]$.
Using the derivation technique discussed in [13], we obtain a fourth order accurate integral formula based on Simpson's $\frac{1}{3}$ rd rule (see Evans [14]).

$$
\begin{aligned}
\int_{x_{k}}^{x_{k+1}} \phi(x) \mathrm{d} x & =\frac{h_{k+1}}{6}\left[\phi_{k}+4 \phi_{k+\frac{1}{2}}+\phi_{k+1}\right], \\
k & =0,1,2, \cdots N
\end{aligned}
$$

where $\phi_{k}=\phi\left(x_{k}\right), \phi_{k+1}=\phi\left(x_{k+1}\right)=\phi\left(x_{k}+h_{k+1}\right)$, $\phi_{k+\frac{1}{2}}=\phi\left(x_{k+\frac{1}{2}}\right)=\phi\left(x_{k}+\frac{h_{k+1}}{2}\right)$, etc.

Then on the variable mesh the value of the integral

$$
\begin{aligned}
\int_{0}^{1} \phi(x) \mathrm{d} x & =\int_{x_{0}}^{x_{1}} \phi(x) \mathrm{d} x+\int_{x_{1}}^{x_{2}} \phi(x) \mathrm{d} x+\cdots+\int_{x_{N}}^{x_{N+1}} \phi(x) \mathrm{d} x \\
& =\sum_{k=0}^{N} \frac{h_{k+1}}{6}\left[\phi_{k}+4 \phi_{k+\frac{1}{2}}+\phi_{k+1}\right]
\end{aligned}
$$

can be found by the repeated application of (4).

Now we discuss the third order numerical method based on arithmetic average discretization for the differential Equation (3).

At the grid point $x_{k}$, we denote

$$
U_{k}^{\prime \prime}=G\left(x_{k}, U_{k}, U_{k}^{\prime}\right) \equiv G_{k} \quad \text { (say), }
$$

and

$$
\alpha_{k}=\left(\frac{\partial G}{\partial u}\right)_{x_{k}}, \beta_{k}=\left(\frac{\partial G}{\partial u^{\prime}}\right)_{x_{k}} .
$$

Using Taylor expansion, from (3), we obtain

$$
\begin{aligned}
& \left(U_{k+1}-\left(1+\sigma_{k}\right) U_{k}+\sigma_{k} U_{k-1}\right) \\
& =\frac{\sigma_{k} h_{k}^{2}}{3}\left[\sigma_{k} G_{k+\frac{1}{2}}+\frac{\left(1+\sigma_{k}\right)}{2} G_{k}+G_{k-\frac{1}{2}}\right]+O\left(h_{k}^{5}\right), \\
& \sigma_{k} \neq 1
\end{aligned}
$$

We need the following approximations:

$$
\begin{aligned}
& \bar{U}_{k+\frac{1}{2}}=\frac{1}{2}\left(U_{k+1}+U_{k}\right), \\
& \bar{U}_{k-\frac{1}{2}}=\frac{1}{2}\left(U_{k-1}+U_{k}\right), \\
& \bar{U}_{k+\frac{1}{2}}^{\prime}=\frac{1}{\sigma_{k} h_{k}}\left(U_{k+1}-U_{k}\right), \\
& \bar{U}_{k-\frac{1}{2}}^{\prime}=\frac{1}{h_{k}}\left(U_{k}-U_{k-1}\right),
\end{aligned}
$$




$$
\bar{U}_{k}^{\prime}=\frac{1}{\sigma_{k}\left(1+\sigma_{k}\right) h_{k}}\left[U_{k+1}-\left(1-\sigma_{k}^{2}\right) U_{k}-\sigma_{k}^{2} U_{k-1}\right]
$$

and let

$$
\bar{G}_{k \pm \frac{1}{2}}=G\left(x_{k \pm \frac{1}{2}}, \bar{U}_{k \pm \frac{1}{2}}, \bar{U}_{k \pm \frac{1}{2}}^{\prime}\right)
$$

It is then easy to see that

$$
\begin{gathered}
\bar{G}_{k+\frac{1}{2}}+\bar{G}_{k-\frac{1}{2}}=2 U_{k}^{\prime \prime}+\frac{h_{k}}{2}\left(\sigma_{k}-1\right) U_{k}^{\prime \prime \prime}+\frac{h_{k}^{2}}{24}\left(1+\sigma_{k}^{2}\right)\left(3 U_{k}^{\prime \prime} \alpha_{k}+U_{k}^{\prime \prime \prime} \beta_{k}+3 U_{k}^{I V}\right)+O\left(h_{k}^{3}\right), \sigma_{k} \neq 1 \\
\bar{G}_{k+\frac{1}{2}}-\bar{G}_{k-\frac{1}{2}}=\frac{h_{k}}{2}\left(1+\sigma_{k}\right) U_{k}^{\prime \prime \prime}+\frac{h_{k}^{2}}{24}\left(\sigma_{k}^{2}-1\right)\left(3 U_{k}^{\prime \prime} \alpha_{k}+U_{k}^{\prime \prime \prime} \beta_{k}+3 U_{k}^{I V}\right)+O\left(h_{k}^{3}\right), \sigma_{k} \neq 1
\end{gathered}
$$

Now, let

$$
\begin{aligned}
& \overline{\bar{U}}_{k}=U_{k}+a h_{k}^{2}\left(\bar{G}_{k+\frac{1}{2}}+\bar{G}_{k-\frac{1}{2}}\right), \\
& \overline{\bar{U}}_{k}^{\prime}=\bar{U}_{k}^{\prime}+b h_{k}\left(\bar{G}_{k+\frac{1}{2}}-\bar{G}_{k-\frac{1}{2}}\right),
\end{aligned}
$$

where " $a$ " and " $b$ " are parameters to be determined.

With the help of (10a) and (10b), from (11a) and (11b), we obtain

$$
\begin{gathered}
\overline{\bar{U}}_{k}=U_{k}+2 a h_{k}^{2} U_{k}^{\prime \prime}+O\left(h_{k}^{3}\right), \sigma_{k} \neq 1, \\
\overline{\bar{U}}_{k}^{\prime}=U_{k}^{\prime}+\frac{h_{k}^{2}}{6}\left[\sigma_{k}+3 b\left(1+\sigma_{k}\right)\right] U_{k}^{\prime \prime \prime}+O\left(h_{k}^{3}\right), \\
\sigma_{k} \neq 1
\end{gathered}
$$

From (9a) and (9b), it follows that

$$
\overline{\bar{G}}_{k}=G_{k}+2 a h_{k}^{2} U_{k}^{\prime \prime} \alpha_{k}+\frac{h_{k}^{2}}{6}\left[\sigma_{k}+3 b\left(1+\sigma_{k}\right)\right] U_{k}^{\prime \prime \prime} \beta_{k}+O\left(h_{k}^{3}\right), \sigma_{k} \neq 1
$$

Then at each internal grid point $x_{k}$, the differential Equation (3) is discretized by

$$
\left(U_{k+1}-\left(1+\sigma_{k}\right) U_{k}+\sigma_{k} U_{k-1}\right)=\frac{\sigma_{k} h_{k}^{2}}{3}\left[\sigma_{k} \bar{G}_{k+\frac{1}{2}}+\frac{\left(1+\sigma_{k}\right)}{2} \overline{\bar{G}}_{k}+\bar{G}_{k-\frac{1}{2}}\right]+\bar{T}_{k}, k=1(1) N
$$

where $\bar{T}_{k}=O\left(h_{k}^{5}\right)$, provided $\sigma_{k} \neq 1$.

Now with the help of the approximations (9a), (9b) and (14), from (6) and (15), we obtain the local truncation error

$$
\bar{T}_{k}=\frac{-h_{k}^{4}}{72} \sigma_{k}\left(1+\sigma_{k}\right)\left[3\left(1-\sigma_{k}+\sigma_{k}^{2}+8 a\right) \alpha_{k} U_{k}^{\prime \prime}+\left(1+\sigma_{k}+\sigma_{k}^{2}+6 b\left(1+\sigma_{k}\right)\right) \beta_{k} U_{k}^{\prime \prime \prime}\right]+O\left(h_{k}^{5}\right), \sigma_{k} \neq 1
$$

The proposed numerical method (15) to be of $O\left(h_{k}^{3}\right)$, the coefficient of $h_{k}^{4}$ in (16) must be zero and we obtain the values of parameters

$$
a=\frac{-\left(1-\sigma_{k}+\sigma_{k}^{2}\right)}{8}, b=\frac{-\left(1+\sigma_{k}+\sigma_{k}^{2}\right)}{6\left(1+\sigma_{k}\right)}
$$

and the local truncation error given by (16) becomes $\bar{T}_{k}=O\left(h_{k}^{5}\right), \quad \sigma_{k} \neq 1$.

\section{Application to Singular Problems}

Consider the linear second order model integro-different- tial equation

$$
\begin{gathered}
\frac{d^{2} u}{d r^{2}}=A(r) \frac{d u}{d r}+B(r) u+f(r)+\int_{0}^{1} K(r, s) \mathrm{d} s, \\
0<r, s<1
\end{gathered}
$$

where $A(r)=\frac{-\alpha}{r}, B(r)=\frac{\alpha}{r^{2}}$. For $\alpha=1$ or 2 , the equation above represents cylindrical or spherical problem, respectively. Replacing the variable $x$ by $r$ and applying the formula (15) to the integro-differential equation (17), we obtain 


$$
\begin{aligned}
& -\left[\sigma_{k} U_{k-1}-\left(1+\sigma_{k}\right) U_{k}+U_{k+1}\right]+\frac{\sigma_{k} h_{k}^{2}}{3}\left[\sigma_{k}\left(A_{k+\frac{1}{2}} \bar{U}_{k+\frac{1}{2}}^{\prime}+B_{k+\frac{1}{2}} \bar{U}_{k+\frac{1}{2}}+f_{k+\frac{1}{2}}+I_{k+\frac{1}{2}}\right)\right. \\
& \left.+\left(A_{k-\frac{1}{2}} \bar{U}_{k-\frac{1}{2}}^{\prime}+B_{k-\frac{1}{2}} \bar{U}_{k-\frac{1}{2}}+f_{k-\frac{1}{2}}+I_{k-\frac{1}{2}}\right)+\left(\frac{1+\sigma_{k}}{2}\right)\left(A_{k} \overline{\bar{U}}_{k}^{\prime}+B_{k} \overline{\bar{U}}_{k}+f_{k}+I_{k}\right)\right]+T_{k}=0, k=1(1) N
\end{aligned}
$$

where

$$
\begin{gathered}
U_{k}=u\left(r_{k}\right), A_{k}=A\left(r_{k}\right), B_{k}=B\left(r_{k}\right), f_{k}=f\left(r_{k}\right), \\
I_{k}=I\left(r_{k}\right)=\int_{0}^{1} K\left(r_{k}, s\right) \mathrm{d} s=\left\{\int_{r_{0}}^{r_{1}}+\int_{r_{1}}^{r_{2}}+\cdots+\int_{r_{N}}^{r_{N+1}}\right\} K\left(r_{k}, s\right) \mathrm{d} s, \\
\overline{\bar{U}}_{k}=U_{k}-\frac{\left(1-\sigma_{k}+\sigma_{k}^{2}\right) h_{k}^{2}}{8}\left[A_{k+\frac{1}{2}} \bar{U}_{k+\frac{1}{2}}^{\prime}+B_{k+\frac{1}{2}} \bar{U}_{k+\frac{1}{2}}+f_{k+\frac{1}{2}}+I_{k+\frac{1}{2}}+A_{k-\frac{1}{2}}^{A_{k-\frac{1}{2}}^{\prime}}+B_{k-\frac{1}{2}} \bar{U}_{k-\frac{1}{2}}+f_{k-\frac{1}{2}}+I_{k-\frac{1}{2}}\right], \\
\overline{\bar{U}}_{k}^{\prime}=\bar{U}_{k}^{\prime}-\frac{\left(1+\sigma_{k}+\sigma_{k}^{2}\right) h_{k}}{6\left(1+\sigma_{k}\right)}\left[A_{k+\frac{1}{2}}^{A_{k+\frac{1}{2}}^{\prime}} \bar{U}_{k+\frac{1}{2}}^{\prime} \bar{U}_{k+\frac{1}{2}}+f_{k+\frac{1}{2}}+I_{k+\frac{1}{2}}-A_{k-\frac{1}{2}}^{A_{k-\frac{1}{2}}^{\prime}} \bar{U}_{k-\frac{1}{2}}^{\prime} \bar{U}_{k-\frac{1}{2}}-f_{k-\frac{1}{2}}-I_{k-\frac{1}{2}}\right],
\end{gathered}
$$

and $T_{k}=O\left(h_{k}^{5}\right)$.

Note that the scheme (18) is directly applicable to singular problem (17) and do not require any fictitious points outside the solution region to compute the scheme. The scheme is also applicable when the internal grid points of the solution region are both even and odd in number as compared to the scheme discussed by Mohanty and Dhall [13] in which the internal grid points are strictly odd in number.

For convergence of the scheme (18), we use the following approximations:

$$
\begin{gathered}
I_{k+\frac{1}{2}}=I_{k}+\frac{\sigma_{k} h_{k}}{2} I_{k}^{\prime}+\frac{\sigma_{k}^{2} h_{k}^{2}}{8} I_{k}^{\prime \prime}+O\left(h_{k}^{3}\right), \\
I_{k-\frac{1}{2}}=I_{k}-\frac{h_{k}}{2} I_{k}^{\prime}+\frac{h_{k}^{2}}{8} I_{k}^{\prime \prime}-O\left(h_{k}^{3}\right), \\
A_{k+\frac{1}{2}}=A_{k}+\frac{\sigma_{k} h_{k}}{2} A_{k}^{\prime}+\frac{\sigma_{k}^{2} h_{k}^{2}}{8} A_{k}^{\prime \prime}+O\left(h_{k}^{3}\right), \\
A_{k-\frac{1}{2}}=A_{k}-\frac{h_{k}}{2} A_{k}^{\prime}+\frac{h_{k}^{2}}{8} A_{k}^{\prime \prime}-O\left(h_{k}^{3}\right) .
\end{gathered}
$$

Similarly, we can define the approximations for $B_{k \pm \frac{1}{2}}$ and $f_{k \pm \frac{1}{2}}$, where

$$
\begin{aligned}
& A_{k}^{\prime}=\frac{d}{d r} A\left(r_{k}\right), B_{k}^{\prime}=\frac{d}{d r} B\left(r_{k}\right), \\
& f_{k}^{\prime}=\frac{d}{d r} f\left(r_{k}\right), K^{\prime}(r, s)=\frac{\partial K}{\partial r}, \cdots \text { etc }
\end{aligned}
$$

and

$$
\begin{aligned}
I_{k}^{\prime} & =\frac{d}{d r} I\left(r_{k}\right)=\int_{0}^{1} K^{\prime}\left(r_{k}, s\right) \mathrm{d} s \\
& =\left\{\int_{r_{0}}^{r_{1}}+\int_{r_{1}}^{r_{2}}+\cdots+\int_{r_{N}}^{r_{N+1}}\right\} K^{\prime}\left(r_{k}, s\right) \mathrm{d} s, \\
I_{k}^{\prime \prime} & =\frac{d^{2}}{d r^{2}} I\left(r_{k}\right)=\int_{0}^{1} K^{\prime \prime}\left(r_{k}, s\right) \mathrm{d} s \\
& =\left\{\int_{r_{0}}^{r_{1}}+\int_{r_{1}}^{r_{2}}+\cdots+\int_{r_{N}}^{r_{N+1}}\right\} K^{\prime \prime}\left(r_{k}, s\right) \mathrm{d} s .
\end{aligned}
$$

Using the approximations (19) in (18), neglecting high order terms and simplifying we get the modified scheme in compact form

$$
\begin{gathered}
{\left[-\sigma_{k}+\operatorname{sub}_{k}\right] U_{k-1}+\left[1+\sigma_{k}+\operatorname{diag}_{k}\right] U_{k}} \\
+\left[-1+\sup _{k}\right] U_{k+1}+\phi_{k}+T_{k}=0
\end{gathered}
$$

where

$$
\begin{aligned}
\operatorname{sub}_{k}= & \frac{-\sigma_{k}^{2} h_{k}}{6} A_{k}-\frac{\sigma_{k} h_{k}}{3}\left[A_{k}-\frac{h_{k}}{2} A_{k}^{\prime}+\frac{h_{k}^{2}}{8} A_{k}^{\prime \prime}\right] \\
& +\frac{\sigma_{k} h_{k}^{2}}{6}\left[B_{k}-\frac{h_{k}}{2} B_{k}^{\prime}\right] \\
& -\frac{\sigma_{k}\left(1+\sigma_{k}+\sigma_{k}^{2}\right) A_{k} h_{k}^{2}}{72}\left[2 A_{k}-h_{k}\left(A_{k}^{\prime}+B_{k}\right)\right] \\
& +\frac{\sigma_{k}\left(1+\sigma_{k}\right)\left(1-\sigma_{k}+\sigma_{k}^{2}\right) A_{k} B_{k} h_{k}^{3}}{48},
\end{aligned}
$$




$$
\begin{aligned}
\operatorname{diag}_{k}= & \frac{-\left(1-\sigma_{k}^{2}\right)}{6} h_{k} A_{k}-\frac{\sigma_{k}\left(1+\sigma_{k}\right) h_{k}^{2}}{6}\left[A_{k}^{\prime}-\frac{\left(1-\sigma_{k}\right) h_{k}}{4} A_{k}^{\prime \prime}\right]+\frac{\sigma_{k}\left(1+\sigma_{k}\right) h_{k}^{2}}{3}\left[B_{k}+\frac{\left(\sigma_{k}-1\right) h_{k} B_{k}^{\prime}}{4}+\frac{\left(1-\sigma_{k}+\sigma_{k}^{2}\right) h_{k}^{2} B_{k}^{\prime \prime}}{8}\right] \\
& +\frac{\left(1+\sigma_{k}\right)\left(1+\sigma_{k}+\sigma_{k}^{2}\right) A_{k} h_{k}^{2}}{36}\left[A_{k}-\frac{\sigma_{k} B_{k}^{\prime} h_{k}^{2}}{2}\right]+\frac{\left(1+\sigma_{k}\right)\left(1-\sigma_{k}+\sigma_{k}^{2}\right) B_{k} h_{k}^{3}}{48}\left[\left(1-\sigma_{k}\right) A_{k}-2 \sigma_{k} B_{k} h_{k}\right], \\
\sup _{k}= & \frac{h_{k}}{6} A_{k}+\frac{\sigma_{k} h_{k}}{3}\left[A_{k}+\frac{\sigma_{k} h_{k}}{2} A_{k}^{\prime}+\frac{\sigma_{k}^{2} h_{k}^{2}}{8} A_{k}^{\prime \prime}\right]+\frac{\sigma_{k}^{2} h_{k}^{2}}{6}\left[B_{k}+\frac{\sigma_{k} h_{k}}{2} B_{k}^{\prime}\right] \\
& -\frac{\left(1+\sigma_{k}+\sigma_{k}^{2}\right) A_{k} h_{k}^{2}}{72}\left[2 A_{k}+\sigma_{k}\left(A_{k}^{\prime}+B_{k}\right) h_{k}\right]-\frac{\left(1+\sigma_{k}\right)\left(1-\sigma_{k}+\sigma_{k}^{2}\right) A_{k} B_{k} h_{k}^{3}}{48}, \\
\phi_{k}= & \frac{\sigma_{k}\left(1+\sigma_{k}\right) h_{k}^{2}}{6}\left[\left(3-\frac{B_{k}\left(1-\sigma_{k}+\sigma_{k}^{2}\right) h_{k}^{2}}{4}\right)\left(f_{k}+I_{k}\right)\right. \\
+ & \left.\left(\sigma_{k}-1-\frac{\left(1+\sigma_{k}+\sigma_{k}^{2}\right) A_{k} h_{k}}{12}\right) h_{k}\left(f_{k}^{\prime}+I_{k}^{\prime}\right)+\frac{\left(1-\sigma_{k}+\sigma_{k}^{2}\right)}{4} h_{k}^{2}\left(f_{k}^{\prime \prime}+I_{k}^{\prime \prime}\right)\right],
\end{aligned}
$$

and $T_{k}=O\left(h_{k}^{5}\right)$.

Incorporating the boundary values $U_{0}=\gamma_{0}, U_{N+1}=\gamma_{1}$, the difference Equation (20) in matrix form can be written as

$$
(\boldsymbol{D}+\boldsymbol{P}) \boldsymbol{U}+\boldsymbol{\phi}+\boldsymbol{T}\left(h_{k}\right)=\mathbf{0}
$$

where $\boldsymbol{D}=\left[-\sigma_{k}, 1+\sigma_{k},-1\right]$ and $\boldsymbol{P}=\left[\operatorname{sub}_{k}, \operatorname{diag}_{k}, \sup _{k}\right]$ are tri-diagonal matrices of order $N$ and

$$
\boldsymbol{\phi}=\left[\phi_{1}+\left(s u b_{1}-\sigma_{1}\right) \gamma_{0}, \phi_{2}, \cdots, \phi_{N-1}, \phi_{N}+\left(\sup _{N}-1\right) \gamma_{1}\right]^{T},
$$

$\boldsymbol{U}=\left[U_{1}, U_{2}, \cdots, U_{N}\right]^{T}, \quad \boldsymbol{T}\left(h_{k}\right)=\left[T_{1}, T_{2}, \cdots, T_{N}\right]^{T}$ and $\mathbf{0}=[0,0, \cdots, 0]^{T}$ are vectors.
Let $\boldsymbol{u}=\left[u_{1}, u_{2}, \cdots, u_{N}\right]^{T} \cong \boldsymbol{U}$ which satisfies

$$
(\boldsymbol{D}+\boldsymbol{P}) \boldsymbol{u}+\boldsymbol{\phi}=\mathbf{0}
$$

Let $e_{k}=u_{k}-U_{k}$ be the discretization error (in the absence of round of errors) at the grid point $r_{k}$ and $\boldsymbol{E}=\boldsymbol{u}-\boldsymbol{U}=\left[e_{1}, e_{2}, \cdots, e_{N}\right]^{T}$ be the error vector.

Subtracting (21) from (22), we obtain the error equation

$$
(\boldsymbol{D}+\boldsymbol{P}) \boldsymbol{E}=\boldsymbol{T}\left(h_{k}\right)
$$

Let $\left|A_{k}\right| \leq G_{1},\left|A_{k}^{\prime}\right| \leq G_{2},\left|A_{k}^{\prime \prime}\right| \leq G_{3},\left|B_{k}\right| \leq H_{1},\left|B_{k}^{\prime}\right| \leq H_{2}$, $\left|B_{k}^{\prime \prime}\right| \leq H_{3}$, where $G_{1}, G_{2}, G_{3}, H_{1}, H_{2}$ and $H_{3}$ are some positive constants. If $p_{i, j}$ be the $(i, j)^{\text {th }}$-element of $\boldsymbol{P}$, then

$$
\begin{gathered}
\left|p_{k, k+1}\right| \leq \frac{h_{k}}{6}\left[\left(1+2 \sigma_{k}\right) G_{1}+\sigma_{k}^{2} h_{k}\left(G_{2}+H_{1}\right)+\frac{\left(1+\sigma_{k}+\sigma_{k}^{2}\right) h_{k}}{6} G_{1}^{2}\right]+O\left(h_{k}^{3}\right), k=1(1) N-1, \\
\left|p_{k, k-1}\right| \leq \frac{\sigma_{k} h_{k}}{6}\left[\left(1+2 \sigma_{k}\right) G_{1}+h_{k}\left(G_{2}+H_{1}\right)+\frac{\left(1+\sigma_{k}+\sigma_{k}^{2}\right) h_{k}}{6} G_{1}^{2}\right]+O\left(h_{k}^{3}\right), k=2(1) N,
\end{gathered}
$$

Thus for sufficiently small $h_{k}$, the matrix $(\boldsymbol{D}+\boldsymbol{P})$ is irreducible (see Varga [15] and Young [16]).
Let $S_{k}$ be the sum of elements of the $k^{\text {th }}$-row of $(\boldsymbol{D}+\boldsymbol{P})$, then

$$
\begin{aligned}
& S_{k}=\sigma_{k}\left[1+\frac{h_{k}}{6}\left(\left(2+\sigma_{k}\right) A_{k}-h_{k} A_{k}^{\prime}\right)+\frac{h_{k}^{2}}{6}\left(\left(2+3 \sigma_{k}\right) B_{k}+\frac{\left(1+\sigma_{k}+\sigma_{k}^{2}\right)}{6} A_{k}^{2}\right)\right]+O\left(h_{k}^{3}\right), k=1 \\
& S_{k}=1-\frac{h_{k}}{6}\left(\left(1+2 \sigma_{k}\right) A_{k}+\sigma_{k}^{2} h_{k} A_{k}^{\prime}\right)+\frac{h_{k}^{2}}{6}\left(\sigma_{k}\left(3+2 \sigma_{k}\right) B_{k}+\frac{\left(1+\sigma_{k}+\sigma_{k}^{2}\right)}{6} A_{k}^{2}\right) O\left(h_{k}^{3}\right), k=N,
\end{aligned}
$$


$S_{k}=\sigma_{k}\left(1+\sigma_{k}\right) \frac{h_{k}^{2}}{2} B_{k}+O\left(h_{k}^{3}\right), k=2(1) N-1$.

Let $G_{1^{*}}=\min _{1 \leq k \leq N}\left|A_{k}\right|, \quad G_{1}^{*}=\max _{1 \leq k \leq N}\left|A_{k}\right|, \quad H_{1^{*}}=\min _{1 \leq k \leq N}\left|B_{k}\right|$, $H_{1}^{*}=\max _{1 \leq k \leq N}\left|B_{k}\right|$, then $0<G_{1^{*}} \leq G_{1} \leq G_{1}^{*}$ and $0<H_{1^{*}} \leq$ $H_{1} \leq H_{1}^{*}$.

It is straightforward to show that for sufficiently small $h_{k},(\boldsymbol{D}+\boldsymbol{P})$ is Monotone (see Varga [15] and Young [16]). Hence $(\boldsymbol{D}+\boldsymbol{P})^{-1}$ exists and $(\boldsymbol{D}+\boldsymbol{P})^{-1} \geq \mathbf{0}$.

From error Equation (23), we have

$$
\|\boldsymbol{E}\| \leq\left\|(\boldsymbol{D}+\boldsymbol{P})^{-1}\right\| \cdot\left\|\boldsymbol{T}\left(h_{k}\right)\right\|
$$

Thus for sufficiently small $h_{k}$, it is easy to show that

$$
\begin{gathered}
S_{k}>\sigma_{k}\left(2+3 \sigma_{k}\right) \frac{h_{k}^{2}}{6} H_{1^{*}}, k=1, \\
S_{k}>\sigma_{k}\left(3+2 \sigma_{k}\right) \frac{h_{k}^{2}}{6} H_{1^{*}}, k=N, \\
S_{k} \geq \sigma_{k}\left(1+\sigma_{k}\right) \frac{h_{k}^{2}}{2} H_{1^{*}}, k=2(1) N-1 .
\end{gathered}
$$

Since

$$
(\boldsymbol{D}+\boldsymbol{P})_{i, k}^{-1} \geq 0 \text { and } \sum_{k=1}^{N}(\boldsymbol{D}+\boldsymbol{P})_{i, k}^{-1} \cdot S_{k}=1, \quad i=1(1) N,
$$

hence

$$
\begin{gathered}
(\boldsymbol{D}+\boldsymbol{P})_{i, k}^{-1} \leq \frac{1}{S_{k}}<\frac{6}{\sigma_{k}\left(2+3 \sigma_{k}\right) H_{1^{*}} h_{k}^{2}}, k=1 \\
(\boldsymbol{D}+\boldsymbol{P})_{i, k}^{-1} \leq \frac{1}{S_{k}}<\frac{6}{\sigma_{k}\left(3+2 \sigma_{k}\right) H_{1^{*}} h_{k}^{2}}, k=N
\end{gathered}
$$

Further,

$$
\begin{aligned}
& \sum_{k=2}^{N-1}(\boldsymbol{D}+\boldsymbol{P})_{i, k}^{-1} \leq \frac{1}{\min _{2 \leq k \leq N-1} S_{k}} \leq \frac{2}{\sigma_{k}\left(1+\sigma_{k}\right) H_{1^{*}} h_{k}^{2}}, \\
& i=1(1) N
\end{aligned}
$$

For any matrix $\boldsymbol{M}$, we define $\|\boldsymbol{M}\|=\max _{1 \leq i \leq N} \sum_{k=1}^{N}\left|m_{i, k}\right|$, where $m_{i, k}$ is the $(i, k)^{\text {th }}$-element of $\boldsymbol{M}$ and $\left\|\boldsymbol{T}\left(h_{k}\right)\right\|=\max _{1 \leq i \leq N}\left|T_{i}\right|$.

With the help of (28a), (28b), (29), from (26), we obtain

$$
\begin{aligned}
\|\boldsymbol{E}\| & \leq \frac{6}{H_{1^{*}} \sigma_{k} h_{k}^{2}}\left(\frac{1}{2+3 \sigma_{k}}+\frac{1}{3\left(1+\sigma_{k}\right)}+\frac{1}{3+2 \sigma_{k}}\right) \cdot O\left(h_{k}^{5}\right) \\
& =O\left(h_{k}^{3}\right)
\end{aligned}
$$

This establishes the third order convergence of the method (20).

\section{Numerical Results}

In this section, we consider another new variable mesh method for the solution of non-linear integro-differential Equation (1) as

$$
\begin{aligned}
& \left(U_{k+1}-\left(1+\sigma_{k}\right) U_{k}+\sigma_{k} U_{k-1}\right) \\
& =\frac{\sigma_{k} h_{k}^{2}}{2}\left[\frac{\left(1+2 \sigma_{k}\right)}{3} \bar{F}_{k+\frac{1}{2}}+\frac{\left(2+\sigma_{k}\right)}{3} \bar{F}_{k-\frac{1}{2}}\right] \\
& +\frac{\sigma_{k} h_{k}^{2}}{2}\left[\frac{\left(1+2 \sigma_{k}\right)}{3} I_{k+\frac{1}{2}}+\frac{\left(2+\sigma_{k}\right)}{3} I_{k-\frac{1}{2}}\right]+O\left(h_{k}^{4}\right), \\
& k=1(1) N
\end{aligned}
$$

where

$$
\begin{aligned}
& \bar{F}_{k+\frac{1}{2}}=F\left(x_{k+\frac{1}{2}}, \bar{U}_{k+\frac{1}{2}}, \bar{U}_{k+\frac{1}{2}}^{\prime}\right), \\
& \bar{F}_{k-\frac{1}{2}}=F\left(x_{k-\frac{1}{2}}, \bar{U}_{k-\frac{1}{2}}, \bar{U}_{k-\frac{1}{2}}^{\prime}\right),
\end{aligned}
$$

and

$$
I_{k}=I\left(x_{k}\right)=\int_{s=0}^{1} K\left(x_{k}, s\right) \mathrm{d} s, k=1(1) N .
$$

The approximations associated with $\bar{F}_{k \pm \frac{1}{2}}$ are already defined by (7a)-(7d). The order of accuracy of the method (31) is of $O\left(h_{k}^{2}\right)$. For evaluating the integral associated with (31), we replace the integral by the trapezoidal rule (see Evans [14]).

$$
\begin{aligned}
\int_{0}^{1} \phi(x) \mathrm{d} x & =\int_{x_{0}}^{x_{1}} \phi(x) \mathrm{d} x+\int_{x_{1}}^{x_{2}} \phi(x) \mathrm{d} x+\cdots+\int_{x_{N}}^{x_{N+1}} \phi(x) \mathrm{d} x \\
& =\sum_{k=0}^{N} \frac{h_{k+1}}{2}\left[\phi_{k}+\phi_{k+1}\right]
\end{aligned}
$$

In this section, we have solved two benchmark problems using the proposed method described by equation (15) and compared our results with those obtained by using the variable mesh method discussed by Mohanty and Dhall [13] only for the cases when internal grid points are odd in number. We have also computed our results using uniform mesh (when $\sigma_{k}=1$ ) for all values of $N$. The boundary conditions may be obtained using the exact solution as a test procedure. The linear difference equation has been solved using a tri-diagonal solver, whereas non-linear difference equations have been solved using the Newton-Raphson method (see Kelly [17] and 
Evans [18]). While using the Newton-Raphson method, the iterations were stopped when absolute error tolerance $\leq 10^{-12}$ was achieved.

The unit interval $[0,1]$ in the space-direction is divided into $(N+1)$ points with

$$
\begin{aligned}
& 0=x_{0}<x_{1}<\cdots x_{N}<x_{N+1}=1, \quad \text { We may write } \\
& \qquad \begin{aligned}
1 & =x_{N+1}-x_{0}=\left(x_{N+1}-x_{N}\right)+\left(x_{N}-x_{N-1}\right)+\cdots+\left(x_{1}-x_{0}\right) \\
& =h_{N+1}+h_{N}+\cdots+h_{1}=\left(1+\sigma_{1}+\sigma_{1} \sigma_{2}+\cdots+\sigma_{1} \sigma_{2} \cdots \sigma_{N}\right) h_{1} .
\end{aligned}
\end{aligned}
$$

For simplicity, we consider $\sigma_{k}=\sigma$ (a constant), $k=$ $1,2, \cdots, N$, then from (33) we have

$$
h_{1}=\frac{1-\sigma}{1-\sigma^{N+1}} .
$$

By prescribing the total number of mesh points to be where

and

$$
h_{k}=x_{k}-x_{k-1}
$$

$$
\sigma_{k}=\left(h_{k+1} / h_{k}\right)>0, k=1,2, \cdots, N .
$$

Example 4.1 $\varepsilon \frac{\mathrm{d}^{2} u}{\mathrm{~d} r^{2}}+\frac{\alpha}{r} \frac{\mathrm{d} u}{\mathrm{~d} r}-\frac{\alpha}{r^{2}} u=\alpha+2 \varepsilon+r\left(r-\frac{\alpha}{\sqrt{\varepsilon}}-4 \sqrt{\varepsilon}\right) \mathrm{e}^{\frac{-r}{\sqrt{\varepsilon}}}-\frac{\alpha+2 \varepsilon}{\sqrt{\varepsilon}} \int_{s=0}^{1} r \mathrm{e}^{\frac{-r s}{\sqrt{\varepsilon}}} \mathrm{d} s, 0<r<1$,

(Linear equation in polar coordinates)

The exact solution is given by $u(r)=r^{2} e^{\frac{-r}{\sqrt{\varepsilon}}}$. The

maximum absolute errors are tabulated in Table $\mathbf{1}$ for various values of $N$.

$\begin{aligned} \text { Example } 4.2 \varepsilon\left(\frac{\mathrm{d}^{2} u}{\mathrm{~d} r^{2}}+\frac{\alpha}{r} \frac{\mathrm{d} u}{\mathrm{~d} r}-\frac{\alpha}{r^{2}} u\right)= & u \frac{\mathrm{d} u}{\mathrm{~d} r}+(2+\alpha) \varepsilon+\frac{r}{\varepsilon^{2}}[r-(4+\alpha) \varepsilon] \mathrm{e}^{\frac{-r}{\varepsilon}} \\ & +\frac{r^{3}}{\varepsilon}(r-2 \varepsilon) \mathrm{e}^{\frac{-2 r}{\varepsilon}}-(2+\alpha) \int_{s=0}^{1} r \mathrm{e}^{\frac{-r s}{\varepsilon}} \mathrm{d} s, 0<r<1,\end{aligned}$

(Model Burger's equation in polar coordinates)

The exact solution is given by $u(r)=r^{2} \mathrm{e}^{\frac{-r}{\varepsilon}}$. The maximum absolute errors are tabulated in Table 2 for various values of $N$.

\section{Final Remarks}

Using three variable mesh points, we have discussed a new numerical methods of accuracy of $O\left(h_{k}^{3}\right)$ based on arithmetic average discretizations for the solution of the non-linear integro-differential Equation (1). Mohanty and Dhall [13] have developed a third order variable mesh method based on Numerov type discretization, which is only applicable to the solution space having odd number of grid points. Although the proposed variable mesh method involve more algebra, but applicable to the solution space having both odd and even number of internal grid points. In addition, the proposed methods are directly applicable to singular problems and we do not require any fictitious points near the boundaries to incorporate the singular point. The numerical results indicate that the proposed method is computationally nearly equal to the method discussed in [13] and applicable to the solution space with all internal grid points. We have tabulated maximum absolute errors for different mesh sizes. Our mesh sizes are either in increasing or in decreasing order. So it is not possible to estimate the order of convergence of the proposed method. Order of convergence can be estimated for uniform mesh using the formula $\log \left(e_{h_{1}} / e_{h_{2}}\right) / \log \left(h_{1} / h_{2}\right)$, where $e_{h_{1}}$ and $e_{h_{2}}$ are the maximum absolute errors for two grid mesh widths $h_{1}$ and $h_{2}$, respectively. For ex: in Table 2, let us consider the case $\alpha=1, \varepsilon=0.01, N=30$ and $N=60$, i.e. $h=\frac{1}{30}=h_{1}$ (say) and $h=\frac{1}{60}=h_{2}$ (say) and the corresponding maximum absolute errors are $0.3116(-8)$ and $0.1978(-9)$, respectively. Using the above formula the 
Table 1. The maximum absolute errors for Example 4.1.

\begin{tabular}{|c|c|c|c|c|c|c|c|c|}
\hline \multirow{3}{*}{$\mathrm{N}$} & \multicolumn{4}{|c|}{$O\left(h_{k}^{3}\right)$-proposed method (20) } & \multicolumn{4}{|c|}{$O\left(h_{k}^{2}\right)$-proposed method $(31)$} \\
\hline & \multicolumn{2}{|c|}{$\alpha=1$} & \multicolumn{2}{|c|}{$\alpha=2$} & \multicolumn{2}{|c|}{$\alpha=1$} & \multicolumn{2}{|c|}{$\alpha=2$} \\
\hline & $\varepsilon=0.1$ & $\varepsilon=0.01$ & $\varepsilon=0.1$ & $\varepsilon=0.01$ & $\varepsilon=0.1$ & $\varepsilon=0.01$ & $\varepsilon=0.1$ & $\varepsilon=0.01$ \\
\hline 20 & $0.2062 \mathrm{E}-4$ & $0.3334 \mathrm{E}-4$ & $0.2528 \mathrm{E}-4$ & $0.2800 \mathrm{E}-3$ & $0.1906 \mathrm{E}-3$ & $0.4772 \mathrm{E}-3$ & $0.2040 \mathrm{E}-3$ & $0.2240 \mathrm{E}-2$ \\
\hline 25 & $\begin{array}{c}0.1519 \mathrm{E}-4 \\
* 0.1416 \mathrm{E}-4\end{array}$ & $\begin{array}{c}0.2511 \mathrm{E}-4 \\
* 0.2405 \mathrm{E}-4\end{array}$ & $\begin{array}{c}0.1810 \mathrm{E}-4 \\
* 0.1723 \mathrm{E}-4\end{array}$ & $\begin{array}{c}0.9455 \mathrm{E}-4 \\
* 0.9376 \mathrm{E}-4\end{array}$ & $0.1665 \mathrm{E}-3$ & $0.1209 \mathrm{E}-3$ & $0.1783 \mathrm{E}-3$ & $0.8935 \mathrm{E}-3$ \\
\hline 30 & $0.1149 \mathrm{E}-4$ & $0.1216 \mathrm{E}-4$ & $0.1491 \mathrm{E}-4$ & $0.4234 \mathrm{E}-4$ & $0.1507 \mathrm{E}-3$ & $0.2864 \mathrm{E}-4$ & $0.1614 \mathrm{E}-3$ & $0.3438 \mathrm{E}-3$ \\
\hline 35 & $\begin{array}{c}0.1082 \mathrm{E}-4 \\
* 0.1022 \mathrm{E}-4\end{array}$ & $\begin{array}{c}0.9128 \mathrm{E}-5 \\
* 0.9075 \mathrm{E}-5\end{array}$ & $\begin{array}{c}0.1348 \mathrm{E}-4 \\
* 0.1289 \mathrm{E}-4\end{array}$ & $\begin{array}{c}0.1661 \mathrm{E}-4 \\
* 0.1618 \mathrm{E}-4\end{array}$ & $0.1402 \mathrm{E}-3$ & $0.2224 \mathrm{E}-4$ & $0.1502 \mathrm{E}-3$ & $0.8741 \mathrm{E}-4$ \\
\hline 40 & $0.1030 \mathrm{E}-4$ & $0.8811 \mathrm{E}-5$ & $0.1199 \mathrm{E}-4$ & $0.1116 \mathrm{E}-4$ & $0.1299 \mathrm{E}-3$ & $0.1599 \mathrm{E}-4$ & $0.1391 \mathrm{E}-3$ & $0.5240 \mathrm{E}-4$ \\
\hline 45 & $\begin{array}{c}0.8989 \mathrm{E}-5 \\
* 0.8910 \mathrm{E}-5\end{array}$ & $\begin{array}{c}0.7612 \mathrm{E}-5 \\
* 0.7588 \mathrm{E}-5\end{array}$ & $\begin{array}{c}0.9217 \mathrm{E}-5 \\
* 0.9148 \mathrm{E}-5\end{array}$ & $\begin{array}{c}0.7880 \mathrm{E}-5 \\
* 0.7823 \mathrm{E}-5\end{array}$ & $0.1238 \mathrm{E}-3$ & $0.1522 \mathrm{E}-4$ & $0.1322 \mathrm{E}-3$ & $0.3338 \mathrm{E}-4$ \\
\hline 50 & $0.8599 \mathrm{E}-5$ & $0.6423 \mathrm{E}-5$ & $0.8875 \mathrm{E}-5$ & $0.6345 \mathrm{E}-5$ & $0.1161 \mathrm{E}-3$ & $0.1451 \mathrm{E}-4$ & $0.1243 \mathrm{E}-3$ & $0.1448 \mathrm{E}-4$ \\
\hline 55 & $\begin{array}{c}0.8110 \mathrm{E}-5 \\
* 0.8066 \mathrm{E}-5\end{array}$ & $\begin{array}{c}0.5666 \mathrm{E}-5 \\
* 0.5612 \mathrm{E}-5\end{array}$ & $\begin{array}{c}0.8420 \mathrm{E}-5 \\
* 0.8366 \mathrm{E}-5\end{array}$ & $\begin{array}{c}0.4529 \mathrm{E}-5 \\
* 0.4488 \mathrm{E}-5\end{array}$ & $0.1112 \mathrm{E}-3$ & $0.1384 \mathrm{E}-4$ & $0.1188 \mathrm{E}-3$ & $0.1360 \mathrm{E}-4$ \\
\hline 60 & $0.7501 \mathrm{E}-5$ & $0.4892 \mathrm{E}-5$ & $0.7977 \mathrm{E}-5$ & $0.2828 \mathrm{E}-5$ & $0.1059 \mathrm{E}-3$ & $0.1325 \mathrm{E}-4$ & $0.1135 \mathrm{E}-3$ & $0.1284 \mathrm{E}-4$ \\
\hline 65 & $\begin{array}{c}0.7123 \mathrm{E}-5 \\
* 0.7070 \mathrm{E}-5\end{array}$ & $\begin{array}{c}0.4088 \mathrm{E}-5 \\
* 0.4024 \mathrm{E}-5\end{array}$ & $\begin{array}{c}0.7362 \mathrm{E}-5 \\
* 0.7316 \mathrm{E}-5\end{array}$ & $\begin{array}{c}0.2524 \mathrm{E}-5 \\
* 0.2487 \mathrm{E}-5\end{array}$ & $0.1022 \mathrm{E}-3$ & $0.1278 \mathrm{E}-4$ & $0.1092 \mathrm{E}-3$ & $0.1252 \mathrm{E}-4$ \\
\hline 70 & $0.6699 \mathrm{E}-5$ & $0.3236 \mathrm{E}-5$ & $0.6886 \mathrm{E}-5$ & $0.2228 \mathrm{E}-5$ & $0.9812 \mathrm{E}-4$ & $0.1227 \mathrm{E}-4$ & $0.1051 \mathrm{E}-3$ & $0.1216 \mathrm{E}-4$ \\
\hline 75 & $\begin{array}{c}0.6128 \mathrm{E}-5 \\
* 0.6084 \mathrm{E}-5\end{array}$ & $\begin{array}{c}0.2422 \mathrm{E}-5 \\
* 0.2392 \mathrm{E}-5\end{array}$ & $\begin{array}{c}0.6332 \mathrm{E}-5 \\
* 0.6304 \mathrm{E}-5\end{array}$ & $\begin{array}{c}0.1907 \mathrm{E}-5 \\
* 0.1888 \mathrm{E}-5\end{array}$ & $0.9512 \mathrm{E}-4$ & $0.1190 \mathrm{E}-4$ & $0.9926 \mathrm{E}-4$ & $0.1182 \mathrm{E}-4$ \\
\hline 80 & $0.5511 \mathrm{E}-5$ & $0.1772 \mathrm{E}-5$ & $0.5834 \mathrm{E}-5$ & $0.1664 \mathrm{E}-5$ & $0.9179 \mathrm{E}-4$ & $0.1148 \mathrm{E}-4$ & $0.9831 \mathrm{E}-4$ & $0.1143 \mathrm{E}-4$ \\
\hline
\end{tabular}

*: Results obtained by using the method discussed in [13].

Table 2. The maximum absolute errors for Example 4.2.

\begin{tabular}{|c|c|c|c|c|c|c|c|c|}
\hline \multirow{3}{*}{$\mathrm{N}$} & \multicolumn{4}{|c|}{$O\left(h_{k}^{3}\right)$-proposed method (15) } & \multicolumn{4}{|c|}{$O\left(h_{k}^{2}\right)$-proposed method (15) [for uniform mesh $\sigma_{k}=1$ ] } \\
\hline & \multicolumn{2}{|c|}{$\alpha=1$} & \multicolumn{2}{|c|}{$\alpha=2$} & \multicolumn{2}{|c|}{$\alpha=1$} & \multicolumn{2}{|c|}{$\alpha=2$} \\
\hline & $\varepsilon=0.1$ & $\varepsilon=0.01$ & $\varepsilon=0.1$ & $\varepsilon=0.01$ & $\varepsilon=0.1$ & $\varepsilon=0.01$ & $\varepsilon=0.1$ & $\varepsilon=0.01$ \\
\hline 10 & $0.3582 \mathrm{E}-5$ & $0.2318 \mathrm{E}-6$ & $0.4054 \mathrm{E}-5$ & $0.2022 \mathrm{E}-6$ & $0.3318 \mathrm{E}-6$ & $0.2421 \mathrm{E}-7$ & $0.3844 \mathrm{E}-6$ & $0.2100 \mathrm{E}-7$ \\
\hline 15 & $\begin{array}{c}0.4972 \mathrm{E}-6 \\
* 0.4818 \mathrm{E}-6\end{array}$ & $\begin{array}{c}0.8959 \mathrm{E}-7 \\
* 0.8872 \mathrm{E}-7\end{array}$ & $\begin{array}{c}0.1422 \mathrm{E}-6 \\
* 0.1366 \mathrm{E}-6\end{array}$ & $\begin{array}{c}0.8889 \mathrm{E}-7 \\
* 0.8801 \mathrm{E}-7\end{array}$ & $0.5756 \mathrm{E}-7$ & $0.6250 \mathrm{E}-8$ & $0.7224 \mathrm{E}-7$ & $0.8181 \mathrm{E}-8$ \\
\hline 20 & $0.1104 \mathrm{E}-6$ & $0.5934 \mathrm{E}-7$ & $0.1951 \mathrm{E}-6$ & $0.3283 \mathrm{E}-7$ & $0.1929 \mathrm{E}-7$ & $0.1412 \mathrm{E}-8$ & $0.1616 \mathrm{E}-7$ & $0.1012 \mathrm{E}-8$ \\
\hline 25 & $\begin{array}{c}0.1525 \mathrm{E}-7 \\
* 0.1488 \mathrm{E}-7\end{array}$ & $\begin{array}{c}0.2785 \mathrm{E}-7 \\
* 0.2740 \mathrm{E}-7\end{array}$ & $\begin{array}{c}0.5790 \mathrm{E}-7 \\
* 0.5720 \mathrm{E}-7\end{array}$ & $\begin{array}{c}0.6503 \mathrm{E}-8 \\
* 0.6472 \mathrm{E}-8\end{array}$ & $0.7782 \mathrm{E}-8$ & $0.9215 \mathrm{E}-9$ & $0.8269 \mathrm{E}-8$ & $0.5440 \mathrm{E}-9$ \\
\hline 30 & $0.4865 \mathrm{E}-8$ & $0.1987 \mathrm{E}-8$ & $0.1354 \mathrm{E}-7$ & $0.2173 \mathrm{E}-8$ & $0.3116 \mathrm{E}-8$ & $0.3812 \mathrm{E}-9$ & $0.3880 \mathrm{E}-8$ & $0.4004 \mathrm{E}-9$ \\
\hline 35 & $\begin{array}{c}0.4133 \mathrm{E}-8 \\
* 0.4096 \mathrm{E}-8\end{array}$ & $\begin{array}{c}0.4344 \mathrm{E}-9 \\
* 0.4312 \mathrm{E}-9\end{array}$ & $\begin{array}{c}0.8218 \mathrm{E}-8 \\
* 0.8176 \mathrm{E}-8\end{array}$ & $\begin{array}{c}0.7437 \mathrm{E}-9 \\
* 0.7414 \mathrm{E}-9\end{array}$ & $0.8868 \mathrm{E}-9$ & $0.5381 \mathrm{E}-10$ & $0.1614 \mathrm{E}-8$ & $0.7818 \mathrm{E}-10$ \\
\hline 40 & $0.3373 \mathrm{E}-8$ & $0.5659 \mathrm{E}-10$ & $0.6534 \mathrm{E}-8$ & $0.2147 \mathrm{E}-9$ & $0.8617 \mathrm{E}-9$ & $0.5733 \mathrm{E}-11$ & $0.9210 \mathrm{E}-9$ & $0.1817 \mathrm{E}-10$ \\
\hline 45 & $\begin{array}{c}0.3008 \mathrm{E}-8 \\
* 0.3001 \mathrm{E}-8\end{array}$ & $\begin{array}{c}0.4234 \mathrm{E}-10 \\
* 0.4228 \mathrm{E}-10\end{array}$ & $\begin{array}{c}0.6166 \mathrm{E}-8 \\
* 0.6122 \mathrm{E}-8\end{array}$ & $\begin{array}{c}0.8872 \mathrm{E}-10 \\
* 0.8845 \mathrm{E}-10\end{array}$ & $0.5417 \mathrm{E}-9$ & $0.3230 \mathrm{E}-11$ & $0.7106 \mathrm{E}-9$ & $0.7117 \mathrm{E}-11$ \\
\hline 50 & $0.2743 \mathrm{E}-8$ & $0.2606 \mathrm{E}-10$ & $0.5657 \mathrm{E}-8$ & $0.5971 \mathrm{E}-10$ & $0.4413 \mathrm{E}-9$ & $0.1006 \mathrm{E}-11$ & $0.4818 \mathrm{E}-9$ & $0.4343 \mathrm{E}-11$ \\
\hline 55 & $\begin{array}{c}0.2174 \mathrm{E}-8 \\
* 0.2170 \mathrm{E}-8\end{array}$ & $\begin{array}{c}0.1918 \mathrm{E}-10 \\
* 0.1911 \mathrm{E}-10\end{array}$ & $\begin{array}{c}0.4828 \mathrm{E}-8 \\
* 0.4810 \mathrm{E}-8\end{array}$ & $\begin{array}{c}0.4815 \mathrm{E}-10 \\
* 0.4804 \mathrm{E}-10\end{array}$ & $0.2830 \mathrm{E}-9$ & $0.8716 \mathrm{E}-12$ & $0.3636 \mathrm{E}-9$ & $0.1156 \mathrm{E}-11$ \\
\hline 60 & $0.1676 \mathrm{E}-8$ & $0.1210 \mathrm{E}-10$ & $0.4138 \mathrm{E}-8$ & $0.4104 \mathrm{E}-10$ & $0.1978 \mathrm{E}-9$ & $0.7677 \mathrm{E}-12$ & $0.2442 \mathrm{E}-9$ & $0.8821 \mathrm{E}-12$ \\
\hline
\end{tabular}

*: Results obtained by using the method discussed in [13]. 
order of the convergence of method can be estimated as 3.97 which is nearly equal to 4.0 . Similarly in other cases, we found that the order of the convergence of the method for uniform mesh case is nearly equal to four.

\section{Acknowledgements}

The authors thank the anonymous reviewers for their constructive suggestions, which greatly improved the standard of the paper.

This research was supported by "The University of Delhi" under research grant No. Dean (R)/R \& D/2011/ 423.

\section{References}

[1] H. B. Keller, "Numerical Methods for Two Point Boundary Value Problems", Blaisdell, London, 1968.

[2] P. J. Davis and P. Rabinowitz, "Method of Numerical Integration," 2nd Edition, Academic Press, New York, 1970.

[3] G. M. Phillips, "Analysis of Numerical Iterative Methods for Solving Integral and Integro-Differential Equations," Computer Journal, Vol. 13, No. 3, 1970, pp. 297-300. doi:10.1093/comjnl/13.3.297

[4] P. Linz, "A Method for Approximate Solution of Linear Integro-Differential Equations," SIAM Journal on $\mathrm{Nu}$ merical Analysis, Vol. 11, No. 1, 1974, pp. 137-144. doi: $10.1137 / 0711014$

[5] V. Lakshmikantham and M. R. M. Rao, "Theory of Integro-Differential Equations," Gordon and Breach, London, 1995.

[6] K. E. Atkinson, "The Numerical Solution of Integral Equations of the Second Kind," Cambridge University Press, Cambridge, 1997. doi:10.1017/CBO9780511626340

[7] R. P. Agarwal and D. O'Regan, "Integral and IntegroDifferential Equations: Theory, Method and Applications," Gordon and Breach, London, 2000.

[8] M. K. Jain, S. R. K. Iyenger and G. S. Subramanyam, "Variable Mesh Methods for the Numerical Solution of Two Point Singular Perturbation Problems," Computer
Methods in Applied Mechanics and Engineering, Vol. 42, 1984, pp. 273-286. doi:10.1016/0045-7825(84)90009-4

[9] R. K. Mohanty, “A Family of Variable Mesh Methods for the Estimates of $(\mathrm{du} / \mathrm{dr})$ and the Solution of Nonlinear Two Point Boundary Value Problems with Singularity," Journal of Computational and Applied Mathematics, Vol. 182, No. 1, 2005, pp. 173-187. doi:10.1016/j.cam.2004.11.045

[10] R. K. Mohanty and N. Khosla, "A Third Order Accurate Variable Mesh TAGE Iterative Method for the Numerical Solution of Two Point Nonlinear Singular Boundary Value Problems," International Journal of Computer Mathematics, Vol. 82, No. 10, 2005, pp. 1261-1273. doi: $10.1080 / 00207160500113504$

[11] R. K. Mohanty and N. Khosla, "Application of TAGE Iterative Algorithms to an Efficient Third Order Arithmetic Average Variable Mesh Discretization for Two Point Non-Linear Boundary Value Problems," Applied Mathematics and Computations, Vol. 172, No. 1, 2006, pp. 148-162. doi:10.1016/j.amc.2005.01.134

[12] R. K. Mohanty, "A Class of Non-Uniform Mesh Three Point Arithmetic Average Discretization for $y^{\prime \prime}=f\left(x, y, y^{\prime}\right)$ and the estimates of $y^{\prime}, "$ Applied Mathematics and Computations, Vol. 183, No. 1, 2006, pp. 477-485. doi:10.1016/j.amc.2006.05.071

[13] R. K. Mohanty and D. Dhall, "Third Order Accurate Variable Mesh Discretization and Application of TAGE Iterative Method for the Non-Linear Two-Point Boundary Value Problems with Homogeneous Functions in Integral Form," Applied Mathematics and Computations, Vol. 215, 2009, pp. 2024-2034. doi:10.1016/j.amc.2009.07.046

[14] G. Evans, "Practical Numerical Integration," John Wiley \& Sons, New York, 1993.

[15] R. S. Varga, "Matrix Iterative Analysis," Springer-Verlag, Berlin, 2000. doi:10.1007/978-3-642-05156-2

[16] D. M. Young, "Iterative Solution of Large Linear Systems," Dover Publication, New York, 2003.

[17] C. T. Kelly, "Iterative Methods for Linear and Non-linear equations," SIAM Publication, Philadelphia, 1995.

[18] D. J. Evans, "Iterative Methods for Solving Nonlinear Two Point Boundary Value Problems," International Journal of Computer Mathematics, Vol. 72, No. 3, 1999, pp. 395-401. doi: 10.1080/00207169908804862 